\title{
Sistematização da assistência de enfermagem com paciente portadora de Síndrome de Guillain-Barré: relato de caso
}

\author{
Systematization of nursing care for a patient with Guillain-Barré syndrome: case report \\ Sistematización de la atención de enfermería a un paciente con síndrome de Guillain- \\ Barré: reporte de caso
}

Thiago Marcírio Gonçalves de Castro ${ }^{1 *}$, José Benedito dos Santos Batista Neto1, Livia Caroline Machado da Silva ${ }^{1}$, Thacyana Vitória Lopes de Carvalho', Daniele Lima dos Anjos Reis ${ }^{1}$, Renata Campos de Sousa Borges ${ }^{1}$, Milena Coelho Fernandes Caldato ${ }^{1}$, José Ronaldo Teixeira de Sousa Júnior², Ismaelino Mauro Nunes Magno², Genislaine Ferreira Pereira ${ }^{3}$.

\section{RESUMO}

Objetivo: Relatar a experiência da aplicação da Sistematização da Assistência de Enfermagem (SAE) em uma paciente com Síndrome de Guillain-Barré. Detalhamentos de Caso: Trata-se de um relato de caso. Aplicou-se a SAE a uma paciente com Síndrome de Guillain-Barré, vivenciada durante o seu acompanhamento quando estava internada em um Hospital, seguindo as etapas da SAE, ou seja, Histórico de Enfermagem, Diagnósticos de Enfermagem, Planejamento de Enfermagem, Implementação e Avaliação da Assistência de Enfermagem. A aplicação desta possibilitou a coleta de dados de forma integral do usuário, como antecedentes mórbidos familiares e pessoais, hábitos de vida, queixas, funções fisiológicas de eliminação, exame físico, o que possibilitou a elaboração de 12 diagnósticos de enfermagem, 19 resultados esperados (divididos em curto e longo prazo), 14 prescrições de enfermagem e ainda, acarretou na realização de uma ação de educação em saúde envolvendo cuidados domiciliares com a utilização de terapias alternativas. Considerações Finais: $O$ estudo conseguiu mostrar a eficiência da SAE no atendimento e acompanhamento de pacientes portadores desta condição.

Palavras-chave: Cuidados de enfermagem, Síndrome de Guillain-Barré, Educação em saúde.

\begin{abstract}
Objective: To report the experience of applying Nursing Care Systematization (NCS) in a patient with GuillainBarré Syndrome. Case Details: This is a case report. NCS was applied to a patient with Guillain-Barré Syndrome, experienced during her follow-up when she was admitted to a Hospital, following the steps of SAE, that is, Nursing History, Nursing Diagnoses, Nursing Planning, Implementation and Evaluation of Nursing Care. The application of this made it possible to collect data in a comprehensive form from the user, such as family and personal morbid antecedents, life habits, complaints, physiological functions of elimination, physical examination, which enabled the elaboration of 12 nursing diagnoses, 19 expected results (divided into short and long term), 14 nursing prescriptions and also led to a health education action involving home care with the use of alternative therapies. Final Considerations: The study was able to show the efficiency of SAE in the care and monitoring of patients with this condition.
\end{abstract}

Keywords: Nursing care, Guillain-Barré syndrome, Health education.

1 Universidade do Estado do Pará (UEPA), Tucuruí - PA. *E-mail: thiagogoncalves_2013@hotmail.com

2 Centro Universitário do Pará (CESUPA), Belém - PA.

${ }^{3}$ Secretaria Municipal de Saúde (SMS), Tucuruí - PA. 
RESUMEN

Objetivo: Relatar la experiencia de aplicar la Sistematización de la Atención de Enfermería (SAE) en un paciente con Síndrome de Guillain-Barré. Detalles del Caso: Este es un reporte de un caso. Se aplicó la Sistematización de la Atención de Enfermería a una paciente con Síndrome de Guillain-Barré, experimentado durante el seguimiento de la paciente ingresada en un Hospital, siguiendo los pasos del SAE, es decir, Historia de Enfermería, Diagnósticos de Enfermería, Planificación, Implementación y Evaluación de la Asistencia de Enfermería en Enfermería. La aplicación del SAE, permitió recolectar datos de manera integral del usuario, tales como antecedentes morbosos familiares, antecedentes morbosos personales, hábitos de vida, quejas, funciones fisiológicas de eliminación, exploración física, lo que permitió la elaboración de 12 diagnósticos de enfermería, 19 resultados esperados (divididos en corto y largo plazo), 14 prescripciones de enfermería y también resultó en una acción de educación en salud que involucró cuidados domiciliarios con el uso de terapias alternativas. Consideraciones Finales: El estudio logró demostrar la eficacia de los SAE en el cuidado y seguimiento de los pacientes con esta patología.

Palabras clave: Atención de enfermería, Síndrome de Guillain-Barré, Educación para la salud.

\section{INTRODUÇÃO}

A Síndrome de Guillain-Barré (SGB) é uma patologia de condição autoimune, enquadra-se como uma neuropatia inflamatória. Possui rápida evolução, ocasionando fraqueza dos membros do indivíduo acometido de forma ascendente, quase sempre simétrica, hipo ou arreflexia e dissociação celuloproteica no líquido cefalorraquidiano. A enfermidade pode chegar à máxima severidade em até 28 dias, com o desenvolvimento de insuficiência respiratória em aproximadamente $1 / 4$ dos casos, a maioria de completa recuperação, mas ainda podem ocorrer sequelas graves e óbitos em até $20 \%$ e $5 \%$ dos casos, respectivamente (NÓBREGA MEB, et al., 2018; WILLISON HJ, et al., 2016).

Sendo uma doença incapacitante, os portadores devem receber atenção redobrada daqueles que realizam sua assistência, principalmente dos profissionais de Enfermagem que lidam, diretamente, com os seus cuidados. Logo, enfermeiros e técnicos devem conhecer os principais sinais e sintomas apresentados, pois a doença tem progressão rápida, tornando-se extremamente grave (CASAROLLI ACG, et al., 2014).

Explica-se que cabe a este profissional o planejamento da assistência, proporcionando atendimento contínuo ao paciente, de maneira sistematizada, exigindo fundamentação teórica para aplicar o raciocínio clínico e a habilidade técnica (SANTOS AG, et al., 2016).

Considera-se o Processo de Enfermagem (PE) como a metodologia utilizada para planejar, implementar e avaliar o cuidado, sendo essencial ao trabalho do enfermeiro (CHAVES LD e SOLAI CA, 2013). Regulamentou-se o PE pela Resolução 358/2009, do Conselho Federal de Enfermagem (COFEN), segundo a qual ele deve ser realizado, de modo deliberado e sistemático, em todos os ambientes onde ocorre a assistência de Enfermagem (COFEN, 2009).

Vê-se o PE como a representação maior do método científico da profissão, direcionado pela SAE, que proporciona o desenvolvimento e a organização do trabalho da equipe pela qual o enfermeiro é responsável (MARIA MA, et al., 2012). A SAE é utilizada para proporcionar um melhor plano e processo de trabalho, objetivando melhoria da assistência e a obtenção de resultados satisfatórios (GUTIÉRREZ MGR e MORAIS SCRV, 2017).

Associado à utilização da SAE, deve ser trabalhada a educação em saúde para estimular o cuidado em casa, assim, serão realizadas as orientações a respeito do autocuidado durante o período de recuperação (BATISTA NETO JBS, et al., 2020). A Educação em Saúde é um processo educativo de construção de conhecimentos em saúde visando o entendimento da população, caracterizada como um conjunto de práticas que contribuem para maior autonomia das pessoas no seu cuidado e no debate com os profissionais e os gestores (FALKENBERG et al., 2014). 
Portanto, sabendo a importância da enfermagem durante os cuidados de pacientes com SGB, o presente estudo teve como objetivo apresentar a aplicação da SAE em uma portadora da SGB, que obteve uma boa resposta, melhorando, consideravelmente, seu quadro clínico.

\section{DETALHAMENTO DO CASO}

Trata-se de um relato de caso sobre a aplicação da SAE a uma paciente com SGB por acadêmicos de enfermagem, sob orientação direta de uma enfermeira-docente, durante a prática curricular obrigatória da disciplina "Metodologia da Assistência de Enfermagem Comunitária e Hospitalar". Ademais, a prática ocorreu em um hospital público do interior do estado do Pará.

A paciente deu entrada no pronto socorro do hospital no dia 03/06/2019 alternando nível de consciência, sendo posteriormente admitida na Unidade de Terapia Intensiva (UTI), onde recebeu o diagnóstico de SGB, com polineuropatia. Ficou internada na UTI por 91 dias, período em que esteve em ventilação mecânica invasiva por tubo orotraqueal e, após, por traqueostomia.

Ademais, foi admitida na Clínica Médica do hospital no dia 06/09/2019, respirando espontaneamente com auxílio de oxigenioterapia, apresentando alterações na força muscular e na fala, comprometendo a capacidade de deambulação, autocuidado, alimentação e comunicação.

Durante sua internação nesse setor, recebeu esquemas de antimicrobianos em razão de pneumonia relacionada à ventilação mecânica. Após tratamento na fase crítica da doença e apresentando melhora do quadro clínico, recebeu alta no dia 30/09/2019, recebendo orientações quanto aos cuidados necessários em sua residência.

\section{$1^{\text {a }}$ etapa - Histórico de Enfermagem}

Para a primeira etapa da SAE, foram realizados a anamnese e o exame físico da paciente, a partir destes, foram verificados os seguintes impasses relacionados a sua saúde: incapacidade de deambular, utilização de acessos venosos e sondas, traqueostomia, presença exacerbada de muco traqueal, taquidispneia, insônia, sentimento de tristeza e saudades da família, impossibilidade de se higienizar sozinha e incapacidade de ingerir alimentos de textura sólida.

\section{2ª etapa - Diagnósticos de Enfermagem}

Consequentemente, tendo noção dos problemas que a paciente possuía, os acadêmicos, utilizando a taxonomia North American Nursing Diagnosis Association (NANDA), iniciaram a elaboração de diagnósticos de enfermagem, que juntamente com a posterior elaboração dos resultados esperados originou o Plano Assistencial da Paciente.

Foram elaborados 12 diagnósticos, classificados por nível de prioridade para que fossem solucionados. Posteriormente, foram elaborados os resultados esperados para a melhora no quadro clínico da paciente. Sabendo que existem três tipos de diagnósticos, os reais ou atuais, os de risco ou potenciais e os de bemestar, foram elaborados resultados esperados de curto prazo (CP) e longo prazo (LP), sendo que os diagnósticos de risco possuíam apenas resultados de LP, pois estes problemas ainda não haviam se instalado, apenas poderiam vir a acontecer.

Finalmente, após a formulação de todos os diagnósticos, da classificação do menos grave para o mais grave e da criação dos resultados esperados, estes foram inseridos no Plano Assistencial da Paciente (Quadro 1). 
Quadro 1 - Plano Assistencial da Paciente.

\begin{tabular}{|c|c|c|}
\hline Problema Identificado & Diagnóstico de Enfermagem & Resultado Esperado \\
\hline $\begin{array}{l}\text { 1) Incapacidade de } \\
\text { deambular }\end{array}$ & $\begin{array}{l}\text { 1) Integridade da pele prejudicada } \\
\text { relacionada à imobilização física e } \\
\text { circulação alterada, evidenciada por } \\
\text { lesão por pressão de estágio II em } \\
\text { região sacra. }\end{array}$ & $\begin{array}{l}\text { 1) CP: paciente deverá apresentar } \\
\text { diminuição de risco de infecção e } \\
\text { exsudato após curativo. } \\
\text { LP: paciente apresentará cicatrização da } \\
\text { lesão. }\end{array}$ \\
\hline $\begin{array}{l}\text { 2) Utilização de } \\
\text { acessos venosos e } \\
\text { sondas }\end{array}$ & $\begin{array}{c}\text { 2) Risco de infecção relacionada à } \\
\text { procedimentos invasivos (por presença } \\
\text { de AV em MSD e Sonda Vesical de } \\
\text { demora). }\end{array}$ & $\begin{array}{l}\text { 2) LP: paciente não desenvolverá } \\
\text { infecção por procedimentos invasivos. }\end{array}$ \\
\hline $\begin{array}{l}\text { 3) Presença } \\
\text { exacerbada de muco } \\
\text { traqueal }\end{array}$ & $\begin{array}{l}\text { 3) Risco de aspiração relacionado à } \\
\text { capacidade prejudicada de deglutir e } \\
\text { presença de Traqueostomia e } \\
\text { secreção mucopurulenta em região } \\
\text { traqueal. }\end{array}$ & $\begin{array}{l}\text { 3) LP: paciente não deverá aspirar } \\
\text { secreção traqueal durante } \\
\text { hospitalização. }\end{array}$ \\
\hline $\begin{array}{l}\text { 4) Autocuidado } \\
\text { prejudicado }\end{array}$ & $\begin{array}{l}\text { 4) Síndrome do Déficit do autocuidado } \\
\text { relacionado à diminuição da força } \\
\text { muscular, secundária à Síndrome de } \\
\text { Guillain-Barré evidenciado por } \\
\text { necessidade de ajuda total para } \\
\text { realização do autocuidado. }\end{array}$ & $\begin{array}{l}\text { 4) CP: paciente receberá ajuda da } \\
\text { equipe de enfermagem para realização } \\
\text { do autocuidado, higiene oral, pavilhão } \\
\text { auricular, couro cabeludo, genitália após } \\
\text { banho no leito. } \\
\text { LP: paciente deverá retomar capacidade } \\
\text { de promover o autocuidado } \\
\text { independente. }\end{array}$ \\
\hline 5) Taquidispneia & $\begin{array}{c}\text { 5) Padrão respiratório ineficaz } \\
\text { relacionado à fadiga da musculatura } \\
\text { respiratória e posicionamento no leito, } \\
\text { caracterizado por taquidispneia (FR: } \\
33 \text { irpm). }\end{array}$ & $\begin{array}{l}\text { 5) CP: paciente deverá referir alívio da } \\
\text { FR após } 20 \text { minutos. } \\
\text { LP: paciente deverá relatar melhora na } \\
\text { respiração após fisioterapia respiratória } \\
\text { e mudança de decúbito. }\end{array}$ \\
\hline $\begin{array}{l}\text { 6) Incapacidade de } \\
\text { deambular }\end{array}$ & $\begin{array}{l}\text { 6) Mobilidade física prejudicada } \\
\text { relacionada à diminuição da força e da } \\
\text { resistência, secundária à alterações } \\
\text { autoimunes (Síndrome de Guillain- } \\
\text { Barré) evidenciado por capacidade } \\
\text { comprometida de mover-se } \\
\text { intencionalmente no ambiente. }\end{array}$ & $\begin{array}{l}\text { 6) CP: paciente deverá receber ajuda da } \\
\text { equipe de enfermagem e fisioterapia } \\
\text { para realização do fortalecimento } \\
\text { muscular dos membros e tratamento } \\
\text { medicamentoso. } \\
\text { LP: paciente deverá referir aumento na } \\
\text { capacidade de deambular. }\end{array}$ \\
\hline 7) Insônia & $\begin{array}{l}\text { 7) Padrão de sono perturbado } \\
\text { relacionado à algia em MMII e frio } \\
\text { evidenciado por relato verbal. }\end{array}$ & $\begin{array}{l}\text { 7) CP: paciente deverá referir alívio das } \\
\text { dores em MMIl e recuperação do } \\
\text { padrão de sono após aumento da } \\
\text { temperatura no quarto durante } \\
\text { hospitalização. }\end{array}$ \\
\hline 8) Saudades da família & $\begin{array}{l}\text { 8) Risco de maternidade prejudicada } \\
\text { relacionada à hospitalização } \\
\text { prolongada e tristeza. }\end{array}$ & $\begin{array}{l}\text { 8) LP: paciente deverá referir ausência } \\
\text { de tristeza }\end{array}$ \\
\hline $\begin{array}{l}\text { 9) Impossibilidade de } \\
\text { se higienizar sozinha }\end{array}$ & $\begin{array}{l}\text { 9) Cavidade oral prejudicada } \\
\text { relacionada à higiene oral inadequada } \\
\text { evidenciada por língua saburrosa e } \\
\text { dentes com sinais de cárie. }\end{array}$ & $\begin{array}{l}\text { 9) CP: paciente deverá receber ajuda } \\
\text { de enfermagem para realização do } \\
\text { autocuidado. } \\
\text { LP: paciente deverá referir aumento na } \\
\text { capacidade do autocuidado } \\
\text { independente. }\end{array}$ \\
\hline $\begin{array}{l}\text { 10) Incapacidade de } \\
\text { ingerir alimentos de } \\
\text { textura sólida }\end{array}$ & $\begin{array}{l}\text { 10) Deglutição prejudicada } \\
\text { relacionada à traqueostomia } \\
\text { evidenciada por dificuldade em ingerir } \\
\text { alimentos sólidos. }\end{array}$ & $\begin{array}{l}\text { 10) CP: paciente deverá referir } \\
\text { melhora parcial na deglutição de } \\
\text { alimentos sólidos. } \\
\text { LP: paciente deverá referir melhora } \\
\text { total na deglutição de alimentos sólidos. }\end{array}$ \\
\hline 11) Traqueostomia & $\begin{array}{l}\text { 11) Comunicação verbal prejudicada } \\
\text { relacionada à traqueostomia } \\
\text { evidenciado por disfasia e dispneia ao } \\
\text { tentar falar. }\end{array}$ & $\begin{array}{l}\text { 11) CP: paciente deverá referir melhora } \\
\text { parcial na fala. } \\
\text { LP: paciente deverá referir melhora } \\
\text { total na fala após fechamento de } \\
\text { traqueostomia. }\end{array}$ \\
\hline 12) Tristeza & $\begin{array}{l}\text { 12) Risco de sentimento de impotência } \\
\text { relacionado à imobilização física. }\end{array}$ & $\begin{array}{l}\text { 12) LP: paciente deverá referir } \\
\text { ausência do sentimento de impotência. }\end{array}$ \\
\hline
\end{tabular}

Fonte: CASTRO TMG, et al., 2020. 


\section{$3^{\text {a }}$ etapa - Planejamento de Enfermagem}

Para alcançar os resultados esperados, foram elaboradas 14 prescrições de enfermagem, pois um mesmo problema poderia ser solucionado com uma ou mais prescrições e vice-versa (Quadro 2). Ademais, para cada prescrição, foi estipulado um horário de realização, objetivando a melhora da paciente através da assistência necessária para executá-las. Importante também explanar que as prescrições foram elaboradas considerando as condições técnicas que o hospital dispõe.

Quadro 2 - Quadro de Prescrição de Enfermagem.

\begin{tabular}{|c|c|}
\hline Prescrição de Enfermagem & Horário \\
\hline $\begin{array}{l}\text { 1. Realizar curativo de lesão por pressão com técnica } \\
\text { asséptica após banho no leito ou quando necessário. }\end{array}$ & Manhã \\
\hline 2. Realizar mudança de decúbito de duas em duas horas. & $8 \mathrm{~h} / 10 \mathrm{~h} / 12 \mathrm{~h} / 14 \mathrm{~h} / 16 \mathrm{~h} / 18 \mathrm{~h} / 20 \mathrm{~h} / 22 \mathrm{~h} / 24 \mathrm{~h}$ \\
\hline $\begin{array}{l}\text { 3. Avaliar necessidade de troca de todos os dispositivos } \\
\text { invasivos (AV e SVF) }\end{array}$ & Manhã, Tarde e Noite \\
\hline 4. Realizar aspiração de Vias aéreas quando necessário. & Manhã, Tarde e Noite \\
\hline 5. Ofertar oxigenoterapia em macronebulização 3L/m. & Manhã, Tarde e Noite \\
\hline $\begin{array}{l}\text { 6. Realizar banho no leito e assistência no autocuidado } \\
\text { com banho, higiene e cuidados com a pele pela manhã } \\
\text { ou quando necessário. }\end{array}$ & Manhã \\
\hline 7. Monitorar Sinais Vitais de $6 / 6$ horas. & $12 \mathrm{~h} / 18 \mathrm{~h} / 24 \mathrm{~h} / 06 \mathrm{~h}$ \\
\hline $\begin{array}{l}\text { 8. Acionar fisioterapeuta para realização de fisioterapia } \\
\text { respiratória } 2 x \text { ao dia. }\end{array}$ & Manhã e Tarde \\
\hline $\begin{array}{l}\text { 9. Acionar fisioterapeuta para terapia com exercício para } \\
\text { deambulação e equilíbrio }\end{array}$ & Manhã e Tarde \\
\hline $\begin{array}{l}\text { 10. Administrar medicação de horário para tratamento da } \\
\text { Síndrome de Guillain-Barré }\end{array}$ & Conforme prescrição médica \\
\hline $\begin{array}{l}\text { 11. Controlar temperatura ambiente para conforto e } \\
\text { melhora do sono }\end{array}$ & Noite \\
\hline 12. Promover envolvimento familiar no tratamento & $\begin{array}{c}15 \mathrm{~h} \\
\text { (Horário estabelecido para visita pelo } \\
\text { próprio hospital) }\end{array}$ \\
\hline $\begin{array}{l}\text { 13. Acionar fonoaudiólogo para terapia de deglutição e } \\
\text { melhora na comunicação }\end{array}$ & Manhã e Tarde \\
\hline 14. Acionar Psicólogo para ouvir ativamente & Manhã e Tarde \\
\hline
\end{tabular}

Fonte: CASTRO TMG, et al., 2020.

\section{4ª etapa - Implementação}

Essa etapa foi posta em prática pela equipe multiprofissional atuante na clínica em que a cliente estava internada. Todos os dias, durante sua estadia no hospital, esses profissionais realizavam as prescrições propostas.

\section{5 etapa - Avaliação}

A avaliação de enfermagem aconteceu de forma sistemática, em conjunto com a etapa anterior, pois, todos os dias, os profissionais registravam a evolução da paciente em seu prontuário, realizando pequenas adaptações nas prescrições, caso necessário.

Após 25 dias na clínica médica, a cliente recebeu alta e regressou para casa. Assim, os acadêmicos realizaram uma visita domiciliar, para uma nova anamnese e exame físico, objetivando realizar uma comparação com os que foram feitos durante a primeira etapa da SAE e assim verificarem como havia sido a recuperação.

Houve melhora significativa no sistema musculoesquelético da paciente, pois ela já conseguia permanecer em posição ortostática, estava sem a cânula de traqueostomia e conseguia se comunicar verbalmente. Ademais, a paciente relatou que os cuidados elaborados pelos acadêmicos e implementados pelos profissionais foram de suma importância para sua recuperação. 
Ainda na visita, realizou-se uma ação de educação em saúde. Foram feitas recomendações sobre cuidados a serem realizados em casa para a manutenção da qualidade de vida da paciente. Estes basearamse nos princípios das Práticas Integrativas e Complementares (PICS), que abrangem um número grande das chamadas terapias alternativas. Por ajudarem no bem-estar de portadores da SGB, foram estudadas e treinadas para que a devida orientação fosse feita sob supervisão da docente responsável, as seguintes PICS: Osteopatia, Quiropraxia, Respiração e Yoga.

Primeiramente, realizou-se um debate com a paciente e seu cônjuge sobre conceito, funcionalidade, finalidade e importância da realização das terapias em casa. Posteriormente, os acadêmicos mostraram a prática de cada terapia, e estimularam a realização delas pelos participantes, assim, foi possível oferecer um conhecimento mais sólido sobre o desempenho das práticas. Após esse processo de educação em saúde, deu-se por encerrado o acompanhamento, bem-sucedido, da paciente.

\section{DISCUSSÃO}

A assistência da paciente durante sua internação hospitalar e na visita domiciliar após sua alta, contribuiu cientificamente, pois ressaltou-se a importância do olhar clínico em um acompanhamento, destacando a importância da SAE na prevenção de agravos e recuperação do indivíduo, seja no ambiente hospitalar ou domiciliar.

Apesar da SAE ser um requerimento legal e auxiliador na prestação de uma assistência eficaz voltada à necessidade dos clientes, ainda são encontradas entre os profissionais de enfermagem problemáticas em torno dela, principalmente na implantação no ambiente hospitalar, em especial na administração pública. Isso ocorre pela falta de interesse e conhecimento do profissional, efetivo pequeno de enfermeiros, além de recursos financeiros para tal (OLIVEIRA MR, et al., 2019).

Diante da complexidade do quadro de SGB, a assistência de enfermagem junto ao trabalho multiprofissional torna-se indispensável, pois a SAE é desenvolvida mediante a metodologia dividida em cinco etapas, visando avaliar a situação saúde-doença e elaborar cuidados de forma individualizada.

A primeira etapa implicou no Histórico de Enfermagem, que discute a respeito da investigação para identificar problemas e necessidades do paciente para determinar seu estado de saúde, isso possibilitou as buscas de informações que contribuíram para a formulação do plano de cuidados individualizado, levando em consideração as limitações da paciente (ALVIM ALS, 2013).

Acredita-se que a escuta qualificada e um olhar integral do sujeito resultam um bom histórico de Enfermagem, facilitando o levantamento dos problemas, visualizando qual plano assistencial realizar acerca de cada problema encontrado, contribuindo para um melhor atendimento (OLIVEIRA YHA, et al., 2019).

A elaboração do diagnóstico de enfermagem é um fato promissor, por trazer a linguagem comum e taxonomia validada; desmistificar o PE e transformar suas etapas em compromisso profissional com o alcance de resultados das intervenções de enfermagem; viabilizar a caracterização dos assuntos cuidativos como estritamente vinculados às respostas dos clientes; possibilitar a expressão da competência dos enfermeiros e a definição de suas responsabilidades profissionais face às respostas da clientela às intervenções (GARCIA TR e NÓBREGA MML, 2019). Levando em consideração essa definição e a dependência que a SGB pode causar, os diagnósticos foram elencados e classificados para atender especificamente cada um dos problemas encontrados como forma de facilitar os cuidados atribuídos à cliente.

O planejamento está estritamente relacionado com a fase anterior, pois, no decorrer da terceira etapa foi possível organizar uma configuração dos mais graves aos menos graves. Tal elaboração foi seguindo a lógica que se os problemas mais importantes fossem estabilizados, os outros poderiam ter uma evolução mais rápida e satisfatória. Assim, essa classificação foi feita baseada nas Necessidades Humanas Básicas, sendo priorizado, inicialmente, a sua locomoção, para ter sua necessidade de estima salvaguardada, devido essa problemática impossibilitar sua realização de atividades habituais (REGIS LFLV e PORTO IS, 2011). Posteriormente, objetivando a necessidade de segurança, foi elencada a presença de procedimentos invasivos em detrimento do risco de agravo do seu quadro atual. 
Quanto ao cuidado e às prescrições de enfermagem, pode-se dizer que houve contribuições positivas. Isso ocorre, pois, o enfermeiro pode contar com ferramentas que incluem comunicação, interação e articulação das dimensões gerenciais e assistenciais e a contribuição da família no processo em domicílio, tornando-se necessária no desenvolvimento das ações para uma recuperação eficiente (TORRES E, et al., 2013).

Durante a implementação do plano de cuidados, percebeu-se a importância da atuação multiprofissional. Devido à finalização da prática obrigatória, os profissionais do hospital ficaram responsáveis pela aplicação das prescrições e contribuíram com seu núcleo específico de prática para uma assistência global ao usuário, sendo caracterizado por uma junção de saberes com algum nível de troca entre os membros (FILHO NCA e SOUZA AMP, 2017).

A avaliação além de ser feita junto da implementação dos cuidados, também foi realizada na residência da paciente, observou-se um grande avanço no que tange à reabilitação das funções motoras, já que ela foi capaz de realizar movimentos com os braços, levando-os até a altura da boca; também conseguia manter-se de pé, sem apoio, por alguns segundos; realizava movimentos de "troca de passo" e deambulava com auxílio de seu marido. Tais movimentos foram vistos como positivos devido à evolução da SGB, que havia levado a paciente a um quadro de tetraparesia.

Suas funções respiratórias também apresentaram um avanço significativo, sendo relatada apenas dispneia quando se posicionava em decúbito ventral. Ademais, foi observado edema nos membros, decorrente da limitação da movimentação corporal e relato de algia em todo corpo.

A paciente informou ter crises de ansiedade devido o sentimento de saudades dos filhos, evidenciando a necessidade de amor, haja vista que ela estava distante durante todo o período de internação, aproximadamente cinco meses; sobre às demais funções fisiológicas não foram evidenciadas alterações, apresentando-se preservadas.

Sabendo que a principal função afetada nos pacientes é a motora, é necessário que sejam incluídas atividades que auxiliem no processo de reabilitação nas ações de cuidado. Dentre essas, podemos citar as PICS como importantes aliadas. Elas estimulam uma melhora física e psicológica, e oferecem melhor qualidade de vida ao paciente, junto ao apoio multiprofissional. Atualmente são reconhecidas pela OMS 20 práticas complementares, dentre elas estão a Quiropraxia, Osteopatia e Respiração Yoga (BARROS NF, et al., 2018).

O enfermeiro tem a possibilidade de habilitar o paciente para desenvolver práticas alternativas através da educação em saúde, que constitui um conjunto de saberes orientados para a prevenção de doenças e promoção da saúde. Trata-se de um recurso onde o conhecimento científico produzido no campo da saúde, intermediado pelos profissionais da área, atinge o cotidiano das pessoas, uma vez que a compreensão dos condicionantes do processo saúde-doença oferece subsídios para a adoção de novos hábitos e condutas de saúde (SOUZA IVB, et al., 2013).

Sabe-se que é o enfermeiro que mantém um contato maior com os pacientes, levando-o a detectar os problemas e desenvolver ações assistenciais. Quando essas práticas são colocadas na assistência do enfermeiro, ele conseguirá visualizar o paciente integralmente, cuidar e assisti-lo de forma holística, facilitando o planejamento das intervenções que serão aplicadas a eles (ALMEIDA JR, et al., 2018).

Assim, o enfermeiro pode usar a Quiropraxia, uma ciência que utiliza ajustes esqueletais, a fim de restabelecer os movimentos artrocinemáticos, reduzir a compressão neural, devolver os micros movimentos normais à coluna, reconectando todos os segmentos do corpo com o cérebro (GAMA CE, et al., 2019).

Além desta, outra atividade que auxilia na reabilitação é a Osteopatia, uma técnica não invasiva, que incide diretamente no sistema musculoesquelético com o propósito de favorecer melhor mecânica articular, gerando efeitos neurofisiológicos influenciando o Sistema Nervoso Autônomo, ocasionando diminuição das dores (GURGEL FFA, et al., 2017).

Nesse contexto, a Quiropraxia e Osteopatia apresentam-se como método aplicável no tratamento fisioterapêutico de indivíduos que sofrem de distúrbios osteomioarticulares que repercutem em dor e prejuízo de funcionalidade. 
Percebe-se o cuidado diferenciado às pessoas acometidas por essa patologia, fazendo-se necessário, sistematizar a assistência de enfermagem, utilizando métodos colaborativos, como as PICS, que reestabeleçam a qualidade de vida desses pacientes junto ao apoio multiprofissional, em especial da fisioterapia para uma recuperação do sistema musculoesquelético mais eficaz.

Diante disso, ressaltaram-se os conhecimentos e experiências atribuídas pelos discentes envolvidos na assistência, reconhecendo nesse panorama a importância da participação, de forma colaborativa com ensino ativo e efetivo da SAE nos diferentes níveis educacionais da enfermagem.

Portanto, os acadêmicos assumem de forma contínua posturas diferenciadas, de modo a promover uma aprendizagem positiva, visando um melhor aprendizado e humanização em todos os processos de cuidar, independente do cliente, patologia ou ambiente em que se fizer necessário estar para desempenhar um bom trabalho, a fim de trazer o empoderamento ao profissional de enfermagem e aos discentes envolvidos.

\section{REFERÊNCIAS}

1. ALMEIDA JR, et al. O enfermeiro frente às práticas integrativas e complementares em saúde na estratégia de saúde da família. Revista Eletrônica Acervo Saúde, 2018; 18: e77.

2. ALVIM ALS. O Processo de Enfermagem e suas Cinco Etapas. Enferm. Foco, 2013; 4(2): 140-141.

3. BARROS NF, et al. Trabalho interprofissional e as Práticas Integrativas e Complementares no contexto da Atenção Primária à Saúde: potenciais e desafios. Rev. Saúde Debate, 2018; 42 (1): 163-173.

4. BATISTA NETO JBS, et al. Construção de tecnologias educativas como forma de educação em saúde para a prevenção da covid-19: relato de experiência. Revista Eletrônica Acervo Saúde, 2020; 12: e3737.

5. CASAROLLI ACG, et al. Assistência de enfermagem na síndrome de guillain-barré: Uma Revisão da Literatura. Revista Contexto Saúde, 2014; 14 (27): 16-22.

6. CHAVES LD, SOLAI CA. Sistematização da Assistência de Enfermagem: considerações teóricas e aplicabilidade. 2nd ed. São Paulo: Martinari, 2013; 160p.

7. COFEN - CONSELHO FEDERAL DE ENFERMAGEM. Resolução COFEN № 358/2009: Sistematização da Assistência de Enfermagem e a implementação do Processo de Enfermagem em ambientes, públicos ou privados, em que ocorre o cuidado profissional de Enfermagem. Brasília: COFEN; 2009.

8. FALKENBERG MB, et al. Educação em saúde e educação na saúde: conceitos e implicações para a saúde coletiva. Ciência \& Saúde Coletiva, 2014; 19(3): 847-852.

9. FILHO NCA, SOUZA AMP. A percepção sobre o trabalho em equipe multiprofissional dos trabalhadores de um Centro de Atenção Psicossocial em Salvador, Bahia, Brasil. Comun. Saúde Educ., 2017; 21(60):63-76.

10. GAMA CE, et al. Efeito da quiropraxia sobre a dor e mobilidade de pacientes com espondiloartrose cervical. Brazilian Journal of health Review, 2019; 2 (3): 1773-87.

11. GARCIA TR, NÓBREGA MML. Simpósio Nacional de Diagnóstico de Enfermagem: construindo um campo de conhecimento para a Enfermagem. Rev Bras Enferm, 2019;72(3):839-47.

12. GURGEL FFA, et al. Reflexões sobre o emprego da osteopatia nas políticas públicas de saúde no Brasil. Rev. Fisioterapia Brasil, 2017; 18(3):374-81.

13. GUTIÉRREZ MGR, MORAIS SCRV. System atization of nursing care and the formation of professional identity. Rev Bras Enferm, 2017; 70 (2): 436-41.

14. MARIA MA, et al. Systematization of nursing care in urgency and emergency services: feasibility of implementation. Rev Bras Enferm, 2012; 65 (2): 297-303.

15. NÓBREGA MEB, et al. Surto de síndrome de Guillain-Barré possivelmente relacionado à infecção prévia pelo vírus Zika, Região Metropolitana do Recife, Pernambuco, Brasil, 2015. Epidemiol. Serv. Saúde, 2018; 27 (2): e2017039.

16. OLIVEIRA YHA, et al. Assistência de enfermagem ao usuário com carcinoma espinocelular. Rev enferm UFPE on line. 2019; 13: e242832.

17. REGIS LFLV, PORTO IS. Necessidades humanas básicas dos profissionais de enfermagem: situações de (in)satisfação no trabalho. Rev Esc Enferm USP, 2011; 45(2):334-41.

18. SANTOS AG, et al. Nursing care to women with intensive care needs during the cycle pregnancy puerpera. Rev Rede Cuidados Saúde, 2016; 10 (1): 1-12.

19. SOUZA IVB, et al. Educação em Saúde e Enfermagem: Revisão Integrativa da Literatura. Rev. Ciênc. Nova Esperança, 2013; 11(1): 112-121.

20. TORRES E, et al. Sistematização da assistência de enfermagem como ferramenta da gerência do cuidado: estudo de caso. Esc Anna Nery, 2011;15 (4): 730-6.

21. WILLISON HJ, et al. Guillain-Barré syndrome. Lancet, 2016; 388 (10045): 717-27. 